\title{
The RFI monitoring systems for the Medicina and the Sardinia Radio Telescopes
}

\author{
Pietro Bolli ${ }^{1}$, Francesco Gaudiomonte, Filippo Messina \\ INAF - Astronomical Observatory of Cagliari \\ Loc. Poggio dei Pini, Strada 54, 09012 Capoterra (CA), Italy \\ E-mail:pbolli@ca.astro.it
}

Roberto Ambrosini, Claudio Bortolotti, Mauro Roma

INAF - Institute of Radio Astronomy

Via Piero Gobetti, 101, 40129 Bologna, Italy

E-mail: ambrosini@ira.inaf.it

\begin{abstract}
We present here two similar RFI monitoring systems now operational at the radio astronomy observatories at Medicina (30 Km East of Bologna) and the Sardinia Radio Telescope (SRT, a $115 \mathrm{GHz}$ fully steerable $64 \mathrm{~m}$ dish, expected to see the first light by the end of 2010, at $35 \mathrm{Km}$ North of Cagliari). Both systems were specifically designed for monitoring the radio spectrum with an extremely wide frequency range, in combination with very high sensitivity and dynamic range. Their frequency coverage spans both over the narrow bands (internationally) allocated to the Radio Astronomy Service as well as across the much wider frequency bands receivable by the advanced radio astronomical receivers operating at both observatories. While these systems are expected to monitor the interferences generated mainly outside our own premises, we are well aware that all modern electronics are susceptible to produce RFI. Then we are also planning to search for any self-interference case originated within our radio observatories. In order to identify the exact location of the RFI source, we make use of a fixed monitoring station, with antennas on top of a tower placed nearby the radio telescope, as well as of a mobile laboratory. This last is composed of a van fully equipped with high performance instrumentation (spectrum analyzers, receivers, extensible antennas, filtered preamplifiers, etc) and their associated ancillary devices (GPS navigators, antenna pointing readouts, autonomous power supplies, living aids, etc). We will describe in details all the RF components and the careful calibration of their electrical characteristics.
\end{abstract}

RFI mitigation workshop

Groningen, the Netherlands

March 29-31, 2010

\footnotetext{
$1 \quad$ Speaker
} 


\section{Introduction}

It is well known that radio astronomical observations are extremely vulnerable to manmade radio frequency interferences (RFI). For this reason, a lot of effort is usually given to the selection of the site suitable to host a new radio astronomical facility. A radio quiet location generally implies: a reasonable shielding offered by mountains surrounding the radio telescope, a rather large distance from heavily populated centers, a good relationship with local telecommunication authorities to avoid the installation of electrically noisy sources too close by. On the other hand, a site that nowadays looks good enough in terms of RFIs for radio astronomical observations will probably not have the same characteristics in thirty-forty years time; the growth and the proliferation of the commercial use of the radio spectrum is indeed unpredictable on the long term scale. Consider for example in the sixties, when the Medicina's site was selected to host the Northern Cross, a transit instrument composed of two perpendicular arms with a total surface area of $30000 \mathrm{~m}^{2}$ observing the sky at $408 \mathrm{MHz}$, operated by the Institute of Radio Astronomy. At that time indeed the Medicina area was "radio quiet” enough at $408 \mathrm{MHz}$ to host a large interferometer requiring a very flat terrain, extensible in the future, when new science discoveries would have made it attractive for an extension upgrade. However a few decades later, the growth of the nearby city, the development of broadcasting, commercial and military applications, and varied electronic personal gadgets have started to deteriorate the radio frequency environment. This pushed the observatory to spend many resources in order to protect the radio frequency bands allocated to the radio astronomical service (RAS). Therefore, a team of technicians was recruited to take care of the RFI issues, monitoring the radio spectrum and reporting official complaints requiring adherence to the Recommendation ITU-RA769 levels of permissible detrimental levels in the RAS bands. Nowadays, their determination, skill and experience lasted for more than thirty years has made possible to preserve almost free of any interference the allocated bands utilized by the Medicina's receivers. This type of experience has been fully integrated in the study of the design criteria and of the hardware specifications, developed for the RFI monitoring system of SRT.

This article is organized as follows: paragraph 2 briefly presents the SRT's technical specifications and the features of the site selected to host the new radio telescope; then the monitoring systems, both fixed and mobile, are described in paragraph 3. Finally, some results of the RFI activity at the SRT's site are listed in paragraph 4.

\section{Sardinia Radio Telescope}

The Sardinia Radio Telescope (SRT, www.srt.inaf.it) is a challenging scientific project managed by the National Institute for Astrophysics and funded by the Italian Ministry of Education and Scientific Research, the Italian Space Agency (ASI), and the Sardinia Regional Government. Its scientific objectives span over various disciplines: Radio Astronomy, Geodynamics, and Deep Space tracking of interplanetary missions, using both single dish and connected network mode as in VLBI. This is made possible by its versatile structure, which is a 
fully steerable, $64 \mathrm{~m}$ diameter parabolic reflector antenna, capable of operating with high efficiency from 0.3 up to $115 \mathrm{GHz}$ [1]. The SRT's optical system is based on a Gregorian configuration with shaped surfaces in order to reduce standing waves between primary and secondary mirrors, and consequently improving also the blockage efficiency. Moreover the active surface, consisting of 1116 actuators behind the panels of the primary mirror, is deigned not only to compensate for both the gravitational and thermal deformations of the mechanical structure, but also to convert the shaped surface of the primary reflector into a true parabolic surface and extend the upper frequency limit of operation from the primary focus.

In order to identify and characterize a proper site where to install the radio telescope, six RFI measurement campaigns, over the last 15 years, have been made with the mobile laboratory available at the Institute of Radio Astronomy. Specifically, the first three campaigns (1992, 1996, and 1997) were addressed to perform RFI monitoring across the whole Sardinia territory. Finally, in the year 2000, after taking into account many other aspects, the site called "Pranu Sanguni” (Latitude: 39d 29m 50s N, Longitude: 9d 14m 40s E), located approximately $35 \mathrm{Km}$ North of Cagliari in the San Basilio municipality, was selected. After then, three further campaigns (2002, 2004, and 2007) were performed to accurately characterize the spectrum environment at the selected site [2]. Since 2007 a specific fixed RFI station is operating at the site and it is continuously monitoring the radio spectrum up to $3 \mathrm{GHz}$.

\section{The RFI monitoring systems}

We want to preserve the quality of radio environment at the radio telescope sites in order to reach the maximum expected sensitivity for a modern radio telescope. At this end we cannot use precious observing time of the Medicina and SRT radio telescopes simply as monitoring devices of RFI events. Therefore, specifically designed hardware has been built to monitor both the frequency bands allocated by ITU to RAS, and the non-RAS bands, now used by our receivers or potentially usable in the future if their level of RF quietness is acceptable with a significant statistical credibility.

In the special case when man-made interferences are detected, either due to intentional or spurious effects, an official report is transmitted to the Territorial Authority of the National Administration. However, such kind of broadband surveys also produce other benefits. For example, the "robustness" design of the receivers can be greatly improved, by knowing in advance the actual levels and spectral distribution of the interfering signals present at that particular site. Also dynamic scheduling of the radioastronomical observations can make use of the statistically significant "lack" of interference at particular times (at nights, weekends). Finally, future mitigation techniques may be optimized by adapting them to the characteristics of the RFI to be excised or flagged. Great attention is also devoted to the generation of autointerference by all types of devices operating at the telescope site.

The scheme of the RFI monitoring systems adopted at SRT and Medicina consists of two different stations: (i) a fixed station with antennas placed on a tower close to the radio telescope, and (ii) a mobile laboratory to move closer to the RFI transmitter. The combination of those facilities allows to have the highest probability in detecting and identifying all kinds of 
possible RFI (wide-band or narrow-band emission, continuous wave or pulsed signal) as well as their characteristics like amplitude, central frequency, bandwidth, type and content of their modulation, azimuth direction, polarization, and finally the exact location of the transmitting source (by using triangulation with the mobile laboratory). The main features of the monitoring systems installed in the two stations are: high versatility, amplitude sensitivity close to the ITURA769 levels, high dynamic range by appropriate filtering, and an almost continuous frequency coverage of the radio spectrum.

\subsection{The fixed station}

While the mobile van allows multiple triangulation measurements, the fixed station can operate automatically unattended in order to guarantee continuous data acquisition and RFI monitoring $24 \mathrm{~h} /$ day. This feature can produce a database statistically significant for all research that may follow. Basically, the fixed stations operating in Medicina and at the SRT can be divided in four main components:

- Antennas: mainly log periodic dipole arrays (LPDA), optimized Yagis, dual-ridge horns. All of them are placed on top of a tower: they can rotate in azimuth and change linear polarization (or are simply tilted by 45 degrees).

- Front-end boxes installed immediately below the antennas: they utilize commercial active and passive microwave components for filtering, switching and pre-amplifying the incoming signals. All electrical specifications are selected after a detailed study of their tradeoffs for sensitivity, robustness, and bandwidths.

- Back-ends: we mainly use spectrum analyzers for the data acquisition and telecommunications receivers and displays for the identification of the interferers. We currently utilize only coaxial cables to feed the back-ends, but we have also plans to substitute them with optical fibers.

- Control system: a PC, various interface cards, rotor and front-end controllers, and software programs take supervision of the whole system.

In Table I, the technical characteristics of the two fixed stations are reported. Actually, the SRT's monitoring system is still preliminary, to be upgraded soon. For monitoring of RFI coming from satellites, the Medicina station is equipped with a reflector antenna $(1.5 \mathrm{~m}$ diameter) supported by an Az-El tracking rotor, with a 45 deg polarized feed covering the frequency band $1-12 \mathrm{GHz}$.

\subsection{The mobile station}

Each radioastronomical station has its own mobile laboratory for RFI measurements (Fig. 1 shows the mobile station of the SRT). Both units have a retractable telescopic mast that can reach a maximum height of $11 \mathrm{~m}$, where antennas, polarizer and front-end boxes can be accommodated. The RF signal is then sent inside the laboratory for data processing by coaxial cables. The upper operational frequency limit is $18 \mathrm{GHz}$ under normal conditions, but with minor modifications it can be extended up to $40 \mathrm{GHz}$. The mobile stations are self-powered by 
the van alternators or auxiliary batteries, in connection with inverters to provide sinusoidal 220 V @ 50 Hertz. The main characteristics of the Medicina and SRT mobile RFI monitoring systems are listed in Table II: bandwidths are limited by passive filters, while device gain/loss and noise figure data are relative to the band pass central frequency. Of course the sensitivity of the RFI monitoring systems cannot reach instantaneously the levels of the ITU-RA769, but the transportability of the mobile laboratory can recover many tens of $\mathrm{dB}$ of sensitivity by moving into the line of sight or very close to the transmitting source.

\begin{tabular}{|c|c|c|}
\hline Features & Medicina & SRT (current /new version ) \\
\hline Tower height & $25 \mathrm{~m}$ & $12 \mathrm{~m} / \mathrm{TBD}$ \\
\hline Max frequency & $2.4 \mathrm{GHz}$ & $3 \mathrm{GHz} / 18 \mathrm{GHz}$ \\
\hline $\begin{array}{l}\text { Antennas available and } \\
\text { frequency coverage }\end{array}$ & $\begin{array}{l}2 \text { LPDA }(80-500 \mathrm{MHz}) \\
2 \text { LPDA }(500-1000 \mathrm{MHz}) \\
2 \text { dual pol. Yagi }(320-332 \mathrm{MHz}) \\
2 \text { dual pol. Yagi }(400-416 \mathrm{MHz}) \\
2 \text { dual pol. Yagi }(606-612 \mathrm{MHz}) \\
1 \text { reflector } 1.2 \mathrm{~m} \text { pol. } 45^{\circ}(1.3-2.4 \mathrm{GHz})\end{array}$ & $\begin{array}{l}1 \text { LPDA }(0.3-5 \mathrm{GHz}) \\
1 \text { LPDA }(1.2-2.5 \mathrm{GHz}) \\
/ \text { TBD }\end{array}$ \\
\hline Rotor & 1 axis for azimuth direction & 2 axis for azimuth direction and polarization \\
\hline $\begin{array}{l}\text { Lenght \& type of } \\
\text { coaxial cable }\end{array}$ & $\begin{array}{l}35 \text { m LCF 1/2” Cu2Y CELLFLEX (max } 3 \\
\text { GHz) }\end{array}$ & $\begin{array}{l}55 \text { m of FSJ4-50B Andrew (max } 10 \mathrm{GHz}) / \\
T B D\end{array}$ \\
\hline Spectrum Analyzer & AGILENT 8562EC & HP 8594 / AGILENT PSA 4446E \\
\hline Receiver & ICOM R9000 & ICOM R9500 \\
\hline
\end{tabular}

Table I - List of main specifications of the fixed monitoring station installed at Medicina and at SRT. The SRT's column reports in italic the parameters for the next generation system. TBD refers to not yet available hardware.

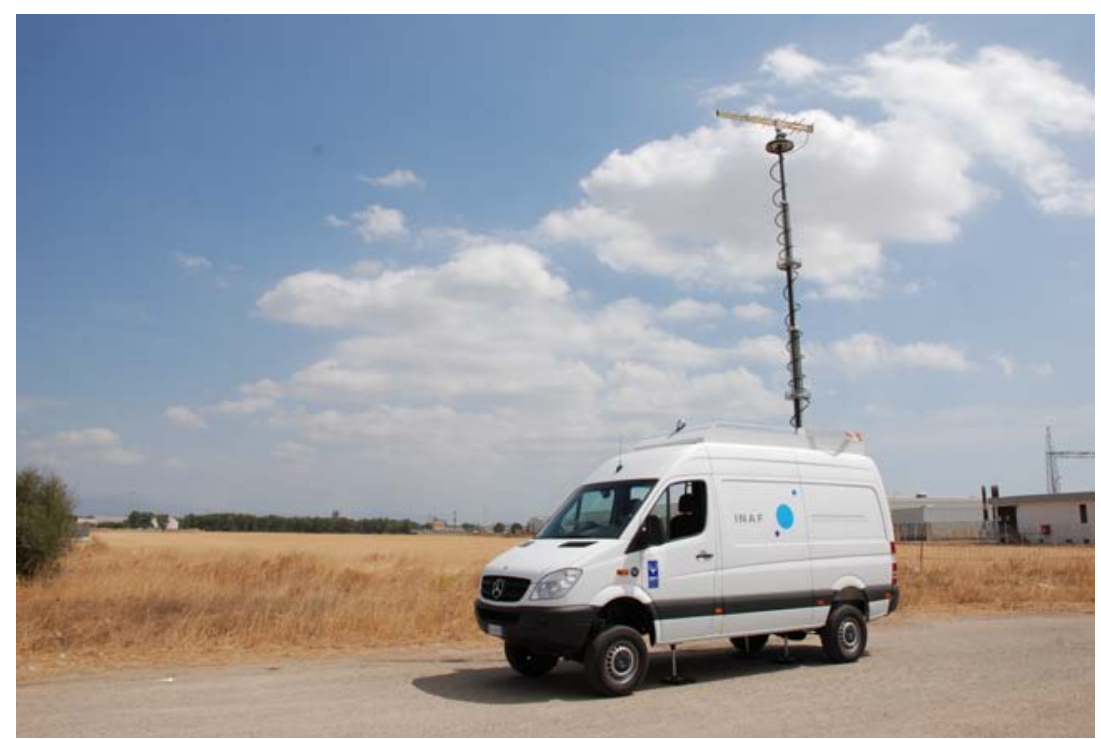

Figure 1 - SRT's mobile station with a log-periodic antenna placed on the pole. 


\begin{tabular}{|c|c|c|c|c|c|c|c|}
\hline \multicolumn{4}{|c|}{ Medicina } & \multicolumn{4}{|c|}{ SRT } \\
\hline $\begin{array}{l}\text { Freq. band @ } \\
-3 \mathrm{~dB}[\mathrm{GHz}]\end{array}$ & $\begin{array}{l}\text { Antenna } \\
\text { gain }\end{array}$ & Chain gain & $\begin{array}{l}\text { Noise } \\
\text { Figure }\end{array}$ & $\begin{array}{l}\text { Freq. band @ } \\
-3 \text { dB [GHz] }\end{array}$ & $\begin{array}{l}\text { Antenna } \\
\text { gain }\end{array}$ & Chain gain & $\begin{array}{l}\text { Noise } \\
\text { Figure }\end{array}$ \\
\hline $0.300-0.434$ & $5.5 \mathrm{dBi}$ & $32.0 \mathrm{~dB}$ & $6.0 \mathrm{~dB}$ & \multirow{2}{*}{$0.290-0.428$} & \multirow{2}{*}{$9.0 \mathrm{dBi}$} & \multirow{2}{*}{$39.1 \mathrm{~dB}$} & \multirow{2}{*}{$4.3 \mathrm{~dB}$} \\
\hline $0.604-0.611$ & $5.5 \mathrm{dBi}$ & $29.0 \mathrm{~dB}$ & $9.5 \mathrm{~dB}$ & & & & \\
\hline $1.22-2.45$ & $4 / 9 \mathrm{dBi}$ & $32.0 \mathrm{~dB}$ & $4.5 \mathrm{~dB}$ & $1.17-1.84$ & $7.0 \mathrm{dBi}$ & $33.9 \mathrm{~dB}$ & $4.2 \mathrm{~dB}$ \\
\hline $2.45-4$ & $4.5 \mathrm{dBi}$ & $26.0 \mathrm{~dB}$ & $5.0 \mathrm{~dB}$ & $1.99-3.37$ & $7.8 \mathrm{dBi}$ & $29.5 \mathrm{~dB}$ & $4.9 \mathrm{~dB}$ \\
\hline $4-8$ & $5.0 \mathrm{dBi}$ & $28.0 \mathrm{~dB}$ & $4.5 \mathrm{~dB}$ & $2.98-5.81$ & $12.0 \mathrm{dBi}$ & $25.2 \mathrm{~dB}$ & $5.4 \mathrm{~dB}$ \\
\hline $8-12$ & $6.0 \mathrm{dBi}$ & $49.0 \mathrm{~dB}$ & $5.0 \mathrm{~dB}$ & $5.22-9.25$ & $11.5 \mathrm{dBi}$ & $48.0 \mathrm{~dB}$ & $6.1 \mathrm{~dB}$ \\
\hline $12-18$ & $8.0 \mathrm{dBi}$ & $37.0 \mathrm{~dB}$ & $8.5 \mathrm{~dB}$ & $7.76-18$ & $12.5 \mathrm{dBi}$ & $40.8 \mathrm{~dB}$ & $6.3 \mathrm{~dB}$ \\
\hline $18-26.5$ & $21.0 \mathrm{dBi}$ & $56.0 \mathrm{~dB}$ & $3.1 \mathrm{~dB}$ & \multirow{2}{*}{$18-40$} & \multirow{2}{*}{ TBD } & \multirow{2}{*}{ TBD } & \multirow{2}{*}{ TBD } \\
\hline $26.5-40$ & $21.0 \mathrm{dBi}$ & $50.5 \mathrm{~dB}$ & $4.0 \mathrm{~dB}$ & & & & \\
\hline
\end{tabular}

Table II - List of the electromagnetic performance of the monitoring systems installed in the mobile laboratories.

\section{RFI monitoring activity at SRT}

So far the RFI monitoring activity has been mainly focused on the hardware set up and technical training of the local personnel. Every week we make by default a full RFI survey with the mobile and the fixed laboratories. All data are recorded and archived. In this context, a preliminary survey of a wind farm has been performed to evaluate the levels of radio frequency emission due to spurious effects associated to the production of electrical energy. Additionally, several RFI measurements were conducted to evaluate the emission of a super-computing facility that will be placed within the SRT premises. The measurements showed that the selfinterference coming from this cluster of computers are not compatible with the ITU-RA769 threshold levels and therefore it has been decided to install a Faraday room to shield those emissions, so to protect the radio telescope [3]

[1] G. Tofani, et al., Status of the Sardinia Radio Telescope Project, in proceedings of SPIE Int. Soc. Opt. Eng. Vol. 7012, 2008.

[2] R. Ambrosini, P. Bolli, C. Bortolotti, F. Messina, M. Roma, La sesta campagna di monitoraggio RFI presso il sito SRT, IRA technical report n. 403/07.

[3] R. Ambrosini, P. Bolli, C. Bortolotti, F. Gaudiomonte, F. Messina, M. Roma, Radio Frequency SelfInterference from a Data Processing Centre at a Radio Telescope site, Experimental Astronomy, vol. 27, 3, pp. 121-130, March, 2010. 